\title{
Standing waves and global existence for nonlinear wave equations with potential, strong, and nonlinear damping terms
}

Wenyi Huang*

\section{"Correspondence:}

huangwy@swufe.edu.cn

Department of Applied

Mathematics, Southwestern

University of Finance and

Economics, Chengdu, 610074,

P.R. China

\begin{abstract}
This paper is concerned with the Cauchy problem of nonlinear wave equations with potential, strong, and nonlinear damping terms. Firstly, by using variational calculus and compactness lemma, the existence of standing waves of the ground states is obtained. Then the instability of the standing wave is shown by applying potential-well arguments and concavity methods. Finally, we show how small the initial data are for the global solutions to exist.
\end{abstract}

Keywords: wave equations; nonlinear damping terms; strong damping terms; global existence; blow-up

\section{Introduction}

Consider the Cauchy problem for nonlinear wave equations with potential, strong, and nonlinear damping terms,

$$
\begin{cases}u_{t t}-\Delta u-\omega \Delta u_{t}+V(x) u+\left|u_{t}\right|^{m-2} u_{t}=|u|^{p-2} u, & \text { in }[0, T) \times R^{n} \\ u(0, x)=u_{0}, \quad u_{t}(0, x)=u_{1}, & \text { in } R^{n},\end{cases}
$$

where $p>2, m \geq 2, T>0, \omega>0$,

$$
u_{0} \in H^{1}\left(R^{n}\right), \quad u_{1} \in L^{2}\left(R^{n}\right),
$$

and

$$
2<p \leq \frac{2 n}{n-2}, \quad \text { for } n \geq 3 ; \quad p>2, \quad \text { for } n \leq 2 .
$$

With the absence of the strong damping term $\Delta u_{t}$, and the damping term $u_{t}$ (see [1]), (1.1) can be viewed as an interaction between one or more discrete oscillators and a field or continuous medium [2].

For the case of linear damping $(\omega=0, m=2)$ and nonlinear sources, Levine [3] showed that the solutions to (1.1) with negative initial energy blow-up for the abstract version. For the nonlinear damping and source terms $(\omega=0, m>2, p>2, V(x)=0)$, the abstract version has been considered by many researchers [4-12]. For instance, Georgiev

\section{Springer}

(c) 2014 Huang; licensee Springer. This is an Open Access article distributed under the terms of the Creative Commons Attribution License (http://creativecommons.org/licenses/by/2.0), which permits unrestricted use, distribution, and reproduction in any medium, provided the original work is properly credited. 
and Todorova [4] prove that if $m \geq p$, a global weak solution exists for any initial data, while if $2<m<p$ the solution blows up in finite time when the initial energy is sufficiently negative. In [5], Todorova considers the additional restriction on $m$. Ikehata [6] considers the solutions of (1.1) with small positive initial energy, using the so-called 'potential-well' theory. The case of strong damping $(\omega>0, m=2, V(x)=0)$ and nonlinear source terms $(p>2)$ has been studied by Gazzola and Squassina in [1]. They prove the global existence of solutions with initial data in the potential well and show that every global solution is uniformly bounded in the natural phase space. Moreover, they prove finite time blow-up for solutions with high energy initial data. However, they do not consider the case of a nonlinear damping term $(\omega>0, m>2, p>2)$.

To the best of our knowledge, little work has been carried out on the existence and instability of the standing wave for (1.1). In this paper, we study the existence of a standing wave with ground state $(\omega=1)$, which is the minimal action solution of the following elliptic equation:

$$
-\Delta \phi+V(x) \phi=|\phi|^{p-2} \phi
$$

Based on the characterization of the ground state and the local well-posedness theory [7], we investigate the instability of the standing wave for the Cauchy problem (1.1). Finally, we derive a sufficient condition of global existence of solutions to the Cauchy problem (1.1) by using the relation between initial data and the ground state solution of (1.4). It should be pointed out that these results in the present paper are unknown to (1.1) before.

For simplicity, throughout this paper we denote $\int_{R^{n}} \cdot d x$ by $\int \cdot d x$ and arbitrary positive constants by $C$.

\section{Preliminaries and statement of main results}

We define the energy space $H$ in the course of nature as

$$
H:=\left\{\varphi \in H^{1}\left(R^{n}\right), \int V(x)|\varphi| d x<\infty\right\} .
$$

By its definition, $H$ is a Hilbert space, continuously embedded in $H^{1}\left(R^{n}\right)$, when endowed with the inner product as follows:

$$
\langle\varphi, \phi\rangle_{H}:=\int(\nabla \varphi \nabla \bar{\phi}+V(x) \varphi \bar{\phi}) d x
$$

whose associated norm is denoted by $\|\cdot\|_{H}$. If $\varphi \in H$, then

$$
\|\varphi\|_{H}=\left(\int|\nabla \varphi|^{2} d x+\int V(x)|\varphi|^{2} d x\right)^{\frac{1}{2}} .
$$

Throughout this paper, we make the following assumptions on $V(x)$ :

$$
\left\{\begin{array}{l}
\inf _{x \in R^{n}} V(x)=\bar{V}(x)>0, \\
V(x) \text { is a } C^{1} \text { bounded measurable function on } R^{n} \\
\lim _{x \rightarrow \infty} V(x)=\infty
\end{array}\right.
$$


According to [1] and [7], we have the following local well-posedness for the Cauchy problem (1.1).

Proposition 2.1 If (1.2) and (1.3) hold, then there exists a unique solution $u(t, x)$ of the Cauchy problem (1.1) on a maximal time interval $[0, T)$, for some $T \in(0, \infty)$ (maximal existence time) such that

$$
\begin{aligned}
& u(t, x) \in C\left([0, T) ; H^{1}\left(R^{n}\right)\right) \cap C^{1}\left([0, T) ; L^{2}\left(R^{n}\right)\right) \cap C^{2}\left([0, T) ; H^{-1}\left(R^{n}\right)\right), \\
& u_{t}(t, x) \in C\left([0, T) ; H^{1}\left(R^{n}\right)\right) \cap L^{m}\left([0, T) \times R^{n}\right),
\end{aligned}
$$

and either $T=\infty$ or $T<\infty$ and $\lim _{t \rightarrow T^{-}}\|u\|_{H^{1}}=\infty$.

Remark 2.2 From Proposition 2.1, it follows that $m=p$ is the critical case, namely for $p \leq m$, a weak solution exists globally in time for any compactly supported initial data; while for $m<p$, blow-up of the solution to the Cauchy problem (1.1) occurs.

We define the functionals

$$
\begin{aligned}
& S(\phi):=\frac{1}{2} \int|\nabla \phi|^{2} d x+\frac{1}{2} \int V(x)|\phi|^{2} d x-\frac{1}{p} \int|\phi|^{p} d x, \\
& R(\phi):=\int|\nabla \phi|^{2} d x+\int V(x)|\phi|^{2} d x-\int|\phi|^{p} d x,
\end{aligned}
$$

for $\phi \in H^{1}\left(R^{n}\right)$, and we define the set

$$
M:=\left\{\phi \in H^{1} \backslash\{0\} ; R(\phi)=0\right\} .
$$

We consider the constrained variational problem

$$
d_{M}:=\inf \left\{\sup _{\lambda \geq 0} S(\lambda \phi): R(\phi)<0, \phi \in H^{1} \backslash\{0\}\right\} .
$$

For the Cauchy problem (1.1), we define unstable and stable sets, $K_{1}$ and $K_{2}$, as follows:

$$
\begin{aligned}
& K_{1} \equiv\left\{\phi \in H^{1}\left(R^{n}\right) \mid R(\phi)<0, S(\phi)<d_{M}\right\}, \\
& K_{2} \equiv\left\{\phi \in H^{1}\left(R^{n}\right) \mid R(\phi)>0, S(\phi)<d_{M}\right\} \cup\{0\} .
\end{aligned}
$$

The main results of this paper are the following.

Theorem 2.3 There exists $Q \in M$ such that

(a1) $S(Q)=\inf _{M} S(\phi)=d_{M}$;

(a2) $Q$ is a ground state solution of (1.4).

From Theorem 2.3, we have the following.

Lemma 2.4 Let $Q(x)$ be the ground state of (1.4). If (1.3) holds, then

$$
S(Q)=\min _{M} S(\phi)
$$


Theorem 2.5 Assume that (1.2)-(1.3) hold and the initial energy E(0) satisfies

$$
\begin{aligned}
E(0) & =\frac{1}{2} \int\left|u_{1}\right|^{2} d x+\frac{1}{2}\left(\int\left|\nabla u_{0}\right|^{2} d x+\int V(x)\left|u_{0}\right|^{2} d x\right)-\frac{1}{p} \int\left|u_{0}\right|^{p} d x \\
& <\frac{p-2}{2 p}\left(\int|\nabla Q|^{2} d x+\int V(x)|Q|^{2} d x\right) .
\end{aligned}
$$

(b1) If $2<m<p$, and there exists $t_{0} \in[0, T)$ such that $u\left(t_{0}\right) \in K_{1}$, then the solution $u(x, t)$ of the Cauchy problem (1.1) blows up in a finite time.

(b2) If $2<p \leq m$, there exists $t_{0} \in[0, T)$ such that $u\left(t_{0}\right) \in K_{2}$, then the solution $u(x, t)$ of the Cauchy problem (1.1) globally exists on $[0, \infty)$. Moreover, for $t \in[0, \infty), u(x, t)$

satisfies

$$
\begin{gathered}
\left\|u_{t}\right\|_{2}^{2}+\frac{p-2}{p}\left(\int\left|\nabla u_{0}\right|^{2} d x+\int V(x)\left|u_{0}\right|^{2} d x\right) \\
<\frac{p-2}{p}\left(\int|\nabla Q|^{2} d x+\int V(x)|Q|^{2} d x\right) .
\end{gathered}
$$

\section{Variational characterization of the ground state}

In this section, we prove Theorem 2.3.

Lemma 3.1 The constrained variational problem

$$
d_{M}:=\inf \left\{\sup _{\lambda \geq 0} S(\lambda \phi): R(\phi)<0, \phi \in H^{1} \backslash\{0\}\right\},
$$

is equivalent to

$$
d_{1}:=\inf \left\{\sup _{\lambda \geq 0} S(\lambda \phi): R(\phi)=0, \phi \in H^{1} \backslash\{0\}\right\}=\inf _{\phi \in M} S(\phi),
$$

and $d_{M}$ provided $2<p \leq \frac{2 n}{n-2}$ as well as $2 \leq m<p$.

Proof Let $\phi \in H^{1}$. Since

$$
S(\lambda \phi)=\frac{\lambda^{2}}{2}\left(\int|\nabla \phi|^{2} d x+\int V(x)|\phi|^{2} d x\right)-\frac{\lambda^{p}}{p} \int|\phi|^{p} d x,
$$

it follows that

$$
\frac{d}{d \lambda} S(\lambda \phi)=\lambda\left(\int|\nabla \phi|^{2} d x+\int V(x)|\phi|^{2} d x\right)-\lambda^{p-1} \int|\phi|^{p} d x .
$$

Thus by $2 \leq m<p$, one sees that there exists some $\lambda_{1} \geq 0$ such that

$$
\sup _{\lambda \geq 0} S(\lambda \phi)=S\left(\lambda_{1} \phi\right)=\lambda_{1}^{2}\left(\frac{1}{2} \int|\nabla \phi|^{2} d x+\frac{1}{2} \int V(x)|\phi|^{2} d x-\frac{\lambda_{1}^{p-2}}{p} \int|\phi|^{p} d x\right),
$$

where $\lambda_{1}$ uniquely depends on $\phi$ and satisfies

$$
\int|\nabla \phi|^{2} d x+\int V(x)|\phi|^{2} d x-\lambda_{1}^{p-2} \int|\phi|^{p} d x=0 .
$$


Since

$$
\frac{d^{2}}{d \lambda^{2}} S(\lambda \phi)=\int|\nabla \phi|^{2} d x+\int V(x)|\phi|^{2} d x-p \lambda^{p-2} \int|\phi|^{p} d x
$$

which together with $p>2$ and (3.6) implies that $\left.\frac{d^{2}}{d \lambda^{2}} S(\lambda \phi)\right|_{\lambda=\lambda_{1}}<0$, we have

$$
\sup _{\lambda \geq 0} S(\lambda \phi)=\frac{p-2}{2 p} \frac{\left(\int|\nabla \phi|^{2} d x+\int V(x)|\phi|^{2} d x\right)^{\frac{p}{p-2}}}{\left(\int|\phi|^{p} d x\right)^{\frac{2}{p-2}}} .
$$

Therefore, the above estimates lead to

$$
\begin{aligned}
d_{M} & =\inf \left\{\sup _{\lambda \geq 0} S(\lambda \phi): R(\phi)<0, \phi \in H^{1} \backslash\{0\}\right\} \\
& =\inf \left\{\frac{p-2}{2 p} \frac{\left(\int|\nabla \phi|^{2} d x+\int V(x)|\phi|^{2} d x\right)^{\frac{p}{p-2}}}{\left(\int|\phi|^{p} d x\right)^{\frac{2}{p-2}}}: R(\phi)<0\right\} .
\end{aligned}
$$

It is easy to see that

$$
\begin{aligned}
d_{1} & =\inf \left\{\sup _{\lambda \geq 0} S(\lambda \phi): R(\phi)=0, \phi \in H^{1} \backslash\{0\}\right\} \\
& =\inf _{\phi \in M}\left\{\frac{p-2}{2 p}\left(\int|\nabla \phi|^{2} d x+\int V(x)|\phi|^{2} d x\right)\right\} .
\end{aligned}
$$

From (2.3)-(2.5), on $M$ one has

$$
S(\phi)=\frac{p-2}{2 p}\left(\int|\nabla \phi|^{2} d x+\int V(x)|\phi|^{2} d x\right) .
$$

It follows that $d_{1}=\inf _{\phi \in M} S(\phi)$ and $S(\phi)>0$ on $M$.

Next we establish the equivalence of the two minimization problems (3.1) and (3.2).

For any $\phi_{0} \in H^{1}$ and $R\left(\phi_{0}\right)<0$, let $\phi_{\beta}(x)=\beta \phi_{0}$. There exists a $\beta_{0} \in(0,1)$ such that $R\left(\phi_{\beta_{0}}\right)=0$, and from (3.8) we get

$$
\begin{aligned}
\sup _{\lambda \geq 0} S\left(\lambda \phi_{\beta_{0}}\right) & =\frac{p-2}{2 p} \frac{\left(\int\left|\nabla \phi_{\beta_{0}}\right|^{2} d x+\int V(x)\left|\phi_{\beta_{0}}\right|^{2} d x\right)^{\frac{p}{p-2}}}{\left(\int\left|\phi_{\beta_{0}}\right|^{p} d x\right)^{\frac{2}{p-2}}} \\
& =\frac{p-2}{2 p} \frac{\beta_{0}^{\frac{2 p}{p-2}}\left(\int\left|\nabla \phi_{0}\right|^{2} d x+\int V(x)\left|\phi_{0}\right|^{2} d x\right)^{\frac{p}{p-2}}}{\beta_{0}^{\frac{2 p}{p-2}}\left(\int\left|\phi_{0}\right|^{p} d x\right)^{\frac{2}{p-2}}} \\
& =\frac{p-2}{2 p} \frac{\left(\int\left|\nabla \phi_{0}\right|^{2} d x+\int V(x)\left|\phi_{0}\right|^{2} d x\right)^{\frac{p}{p-2}}}{\left(\int\left|\phi_{0}\right|^{p} d x\right)^{\frac{2}{p-2}}} .
\end{aligned}
$$

Consequently the two minimization problems (3.8) and (3.9) are equivalent, that is, (3.1) and (3.2) are equivalent. 
Finally, we prove $d_{M}>0$ by showing $d_{1}>0$ in terms of the above equivalence. Since $2<p \leq \frac{2 n}{n-2}$, the Sobolev embedding inequality yields

$$
\int|\phi|^{p} d x \leq C\left(\int|\nabla \phi|^{2} d x+\int V(x)|\phi|^{2} d x\right)^{\frac{p}{2}}
$$

From $R(\phi)=0$, it follows that

$$
\begin{aligned}
& \int|\nabla \phi|^{2} d x+\int V(x)|\phi|^{2} d x \\
& =\int|\phi|^{p} d x \leq C\left(\int|\nabla \phi|^{2} d x+\int V(x)|\phi|^{2} d x\right)^{\frac{p}{2}},
\end{aligned}
$$

which together with $p>2$ implies

$$
\int|\nabla \phi|^{2} d x+\int V(x)|\phi|^{2} d x \geq C>0 .
$$

Therefore, from (3.10), we get

$$
S(\phi) \geq C>0, \quad \phi \in M
$$

Thus from the equivalence of the two minimization problems (3.1) and (3.2) one concludes that $d_{M}>0$ for $2<p \leq \frac{2 n}{n-2}$.

This completes the proof of Lemma 3.1.

Proposition 3.2 $S$ is bounded below on $M$ and $d_{M}>0$.

Proof From (2.3)-(2.6), on $M$ one has

$$
S(\phi)=\frac{p-2}{2 p}\left(\int|\nabla \phi|^{2} d x+\int V(x)|\phi|^{2} d x\right) .
$$

It follows that $S(\phi)>0$ on $M$. So $S$ is bounded below on $M$. From (2.6) we have $d_{M}>0$.

Proposition 3.3 Let $\phi_{\lambda}(x)=\lambda \phi(x)$, for $\phi \in H^{1} \backslash\{0\}$ and $\lambda>0$. Then there exists a unique $\mu>0$ (depending on $\phi$ ) such that $R\left(\phi_{\mu}\right)=0$. Moreover,

$$
R\left(\phi_{\lambda}\right)>0, \quad \text { for } \lambda \in(0, \mu) ; \quad R\left(\phi_{\lambda}\right)<0, \quad \text { for } \lambda \in(\mu, \infty) ;
$$

and

$$
S\left(\phi_{\mu}\right) \geq S\left(\phi_{\lambda}\right), \quad \forall \lambda>0 .
$$

Proof By (2.3) and (2.4), we have

$$
S\left(\phi_{\lambda}\right)=\frac{\lambda^{2}}{2}\left(\int|\nabla \phi|^{2} d x+\int V(x)|\phi|^{2} d x\right)-\frac{\lambda^{p}}{p} \int|\phi|^{p} d x,
$$




$$
R\left(\phi_{\lambda}\right)=\lambda^{2}\left(\int|\nabla \phi|^{2} d x+\int V(x)|\phi|^{2} d x\right)-\lambda^{p} \int|\phi|^{p} d x .
$$

From the definition of $M$, there exists a unique $\mu>0$ such that $R\left(\phi_{\mu}\right)=0$. Moreover,

$$
R\left(\phi_{\lambda}\right)>0, \quad \text { for } \lambda \in(0, \mu) ; \quad R\left(\phi_{\lambda}\right)<0, \quad \text { for } \lambda \in(\mu, \infty) .
$$

Since

$$
\frac{d}{d \lambda} S\left(\phi_{\lambda}\right)=\lambda^{-1} R\left(\phi_{\lambda}\right)
$$

and $R\left(\phi_{\mu}\right)=0$, we have $S\left(\phi_{\mu}\right) \geq S\left(\phi_{\lambda}\right), \forall \lambda>0$.

Next, we solve the variational problem (2.6).

We first give a compactness lemma in [8].

Lemma 3.4 Let $1 \leq p<\frac{N+2}{N-2}$ when $N \geq 3$ and $1 \leq p<\infty$ when $N=1$,2. Then the embedding $H \hookrightarrow L^{p+1}$ is compact.

In the following, we prove Theorem 2.3.

Proof of Theorem 2.3 According to Proposition 3.2, we let $\left\{\phi_{n}, n \in N\right\} \subset M$ be a minimizing sequence for (2.6), that is,

$$
R\left(\phi_{n}\right)=0, \quad S\left(\phi_{n}\right) \rightarrow d_{M} .
$$

From (3.15) and (3.16), we know $\left\|\nabla \phi_{n}\right\|_{2}^{2}$ is bounded for all $n \in N$. Then there exists a subsequence $\left\{\phi_{n_{k}}, k \in N\right\} \subset\left\{\phi_{n}, n \in N\right\}$, such that

$$
\left\{\phi_{n_{k}}\right\} \rightarrow \phi_{\infty} \quad \text { weakly in } H^{1} .
$$

For simplicity, we still denote $\left\{\phi_{n_{k}}, k \in N\right\}$ by $\left\{\phi_{n}, n \in N\right\}$. So we have

$$
\phi_{n} \rightarrow \phi_{\infty} \quad \text { weakly in } H^{1} \text {. }
$$

By Lemma 3.4, we have

$$
\begin{array}{ll}
\phi_{n} \rightarrow \phi_{\infty} & \text { strongly in } L^{2}\left(R^{n}\right), \\
\phi_{n} \rightarrow \phi_{\infty} & \text { strongly in } L^{p}\left(R^{n}\right) .
\end{array}
$$

Next, we prove that $\phi_{\infty} \neq 0$ by contradiction. If $\phi_{\infty} \equiv 0$, from (3.18) and (3.19), we have

$$
\begin{array}{ll}
\phi_{n} \rightarrow 0 & \text { strongly in } L^{2}\left(R^{n}\right), \\
\phi_{n} \rightarrow 0 & \text { strongly in } L^{p}\left(R^{n}\right) .
\end{array}
$$

Since $\phi_{n} \in M, R\left(\phi_{n}\right)=0$, we obtain

$$
\left(\int|\nabla \phi|^{2} d x+\int V(x)|\phi|^{2} d x\right) \rightarrow 0, \quad n \rightarrow \infty .
$$


On the other hand, from (2.3) we have

$$
\left(\int|\nabla \phi|^{2} d x+\int V(x)|\phi|^{2} d x\right)-\frac{2}{p} \int\left|\phi_{n}\right|^{p} d x \rightarrow d_{M} .
$$

From (3.20)-(3.21) we obtain $\left(\int|\nabla \phi|^{2} d x+\int V(x)|\phi|^{2} d x\right) \rightarrow d_{M}$. According to Proposition 3.2, $d_{M} \geq c>0$. This is in contradictory to (3.22). Thus $\phi_{\infty} \neq 0$.

According to Proposition 3.3, we take $Q=\left(\phi_{\infty}\right)_{\mu}$ with $\mu>0$ uniquely determined by the condition $R(Q)=R\left[\left(\phi_{\infty}\right)_{\mu}\right]=0$. From (3.17)-(3.19), we have

$$
\begin{aligned}
& \left(\phi_{\infty}\right)_{\mu} \rightarrow Q \quad \text { strongly in } L^{2}\left(R^{n}\right), \\
& \left(\phi_{\infty}\right)_{\mu} \rightarrow Q \quad \text { strongly in } L^{p}\left(R^{n}\right), \\
& \left(\phi_{\infty}\right)_{\mu} \rightarrow Q \quad \text { weakly in } H^{1}\left(R^{n}\right) .
\end{aligned}
$$

Since $R\left(\phi_{n}\right)=0$ and by Proposition 3.3, we get

$$
S\left[\left(\phi_{\infty}\right)_{\mu}\right] \leq S\left(\phi_{n}\right)
$$

From (3.23)-(3.26), we have

$$
S(Q) \leq \varliminf_{n \rightarrow \infty} S\left[\left(\phi_{\infty}\right)_{\mu}\right] \leq \lim _{n \rightarrow \infty} S\left(\phi_{n}\right)=\inf _{M} S
$$

Since $Q \neq 0$ and $R(Q)=0$, then $Q \in M$. Therefore from (3.27), $Q$ solves the minimization problem

$$
S(Q)=\min _{\phi \in M} S(\phi)
$$

Thus we complete the proof of (a1) of Theorem 2.3.

Next we prove (a2) of Theorem 2.3.

Since $Q$ is a solution of the problem (3.28), there exists a Lagrange multiplier $\Lambda$ such that

$$
\delta_{Q}[S+\Lambda R]=0
$$

where $\delta_{\phi} G$ denotes the variation of $G(\phi)$ about $\phi$. By the formula

$$
\delta_{\phi} G(\phi)=\left.\frac{\partial}{\partial \eta} G(\phi+\eta \delta \phi)\right|_{\eta=0}
$$

we have

$$
\begin{aligned}
\delta_{\phi}[S+\Lambda R]= & (1+2 \Lambda) \int(-\Delta \phi \cdot \delta \phi+V(x) \phi \cdot \delta \phi) d x \\
& -(1+p \Lambda) \int \phi|\phi|^{p-2} \delta \phi d x
\end{aligned}
$$


where $\delta \phi$ denotes the variation of $\phi$. From (3.29), we get

$$
(1+2 \Lambda) \int\left(|\nabla Q|^{2}+V(x)|Q|^{2}\right) d x=(1+p \Lambda) \int|Q|^{p} d x
$$

From $R(Q)=0$, we have

$$
\int\left(|\nabla Q|^{2}+V(x)|Q|^{2}\right) d x=\int|Q|^{p} d x
$$

From (3.31) and (3.32), we obtain $\Lambda=0$. Thus from (3.29) and (3.30), we have

$$
-\Delta Q+V(x) Q=|Q|^{p-2} Q
$$

This indicates that $Q$ is a solution of (1.4) as (1.4) is the Euler Lagrange equation of the functional $S$. Thus we prove (a2) of Theorem 2.3.

So far, we have completed the proof of Theorem 2.3.

\section{Blow-up and global existence}

In this section, we prove Theorem 2.5.

Lemma 4.1 Let $E(0)<d_{M}$, then $K_{1}$ and $K_{2}$ are invariant under the flow generated by (1.1).

Proof Suppose that $u_{0} \in K_{1}$ and $u(t)$ be the solution of (1.1). From Pucci and Serrin [10] and (2.4), we have

$$
\begin{aligned}
S(u(t)) & \leq E(t)=E(0)-\int_{0}^{t}\left\|u_{t}(s, \cdot)\right\|_{m}^{m} d s-\int_{0}^{t}\left\|\nabla u_{t}(s, \cdot)\right\|_{2}^{2} d s \\
& <S(Q)=d_{M}, \quad t \in[0, T) .
\end{aligned}
$$

To prove $u(t) \in K_{1}$, we need to prove

$$
R(u(t))<0, \quad t \in[0, T) .
$$

If (4.1) is not true, by continuity, there would exist a $\bar{t}>0$ such that $R(u(\bar{t}))=0$ because $R\left(u_{0}\right)<0$. It follows that $u(\bar{t}) \in M$. This is impossible because $S(u(\bar{t}))<S(Q)$ and $S(Q)=$ $\min _{\phi \in M} S(\phi)$. Thus (4.1) is true. So $K_{1}$ is invariant under the flow generated by (1.1).

Similarly, we can show that $K_{2}$ is also invariant under the flow generated by (1.1). This completes the proof of Lemma 4.1.

Lemma 4.2 Let the initial data $u_{0}, u_{1}$ satisfy (1.2) and let $u(t, x)$ be a local solution of the Cauchy problem (1.1) on $[0, T)$. If there exists $a u_{0} \in K_{1}$ and $E(0)<d_{M}$, then the inequality

$$
\int\left(|\nabla u|^{2}+V(x)|u|^{2}\right) d x>\frac{2 p}{p-2} d_{M}
$$

is fulfilled for $t \in[0, T)$. 
Proof According to Lemma 4.1, we have $\int|u|^{p} d x>\int\left(|\nabla u|^{2}+V(x)|u|^{2}\right) d x$ for $t \in[0, T)$. Using the above inequality and the identity (2.3), we get

$$
d_{M} \leq \frac{p-2}{2 p} \int\left(|\nabla u|^{2}+V(x)|u|^{2}\right) d x
$$

which completes the proof of Lemma 4.2.

Next, we prove Theorem 2.5.

Proof of Theorem 2.5 By (2.7), we have $S\left(u_{0}\right) \leq E(0)<S(Q)$.

Firstly, we prove (b1) of Theorem 2.5. From the energy identity we have

$$
\int_{0}^{t}\left\|u_{t}(s, \cdot)\right\|_{m}^{m} d s+\int_{0}^{t}\left\|\nabla u_{t}(s, \cdot)\right\|_{2}^{2} d s=E(0)-E(t) \leq d_{M},
$$

for all $t \geq 0$.

Denoting $J(t)=\|u(t, \cdot)\|_{2}^{2}$, we have

$$
\begin{aligned}
J^{\prime \prime}(t)= & 2\left\|u_{t}(t, \cdot)\right\|_{2}^{2}-2 R(u(t))-2 \int \nabla u_{t}(t, x) \nabla u(t, x) d x \\
& -2 \int u(t, x) u_{t}(t, x)\left|u_{t}(t, x)\right|^{m-2} d x .
\end{aligned}
$$

Using the Hölder inequality and the interpolation inequality, we obtain

$$
\begin{aligned}
\left.\left|\int u(t, x) u_{t}(t, x)\right| u_{t}(t, x)\right|^{m-2} d x \mid & \leq\|u(t, \cdot)\|_{m}\left\|u_{t}(t, \cdot)\right\|_{m}^{m-1} \\
& \leq\|u(t, \cdot)\|_{2}^{\delta}\|u(t, \cdot)\|_{p}^{1-\delta}\left\|u_{t}(t, \cdot)\right\|_{m}^{m-1}
\end{aligned}
$$

with $\delta=\frac{\frac{1}{m}-\frac{1}{p}}{\frac{1}{2}-\frac{1}{p}}$. From $R(u)<0$, we have

$$
\int V(x)|u|^{2} d x<\|u\|_{p}^{p}
$$

which together with Lemma 4.1 give

$$
\|u(t, \cdot)\|_{2}^{\delta}\|u(t, \cdot)\|_{p}^{1-\delta}\left\|u_{t}(t, \cdot)\right\|_{m}^{m-1} \leq C\left\|u_{t}(t, \cdot)\right\|_{m}^{m-1}\|u(t, \cdot)\|_{p}^{1-\frac{p}{m}-\delta+\frac{p \delta}{2}}\|u(t, \cdot)\|_{p}^{\frac{p}{m}} .
$$

Using the Young inequality and $1-\frac{p}{m}-\delta+\frac{p \delta}{2}=0$, we have

$$
\begin{gathered}
\|u(t, \cdot)\|_{2}^{\delta}\|u(t, \cdot)\|_{p}^{1-\delta}\left\|u_{t}(t, \cdot)\right\|_{m}^{m-1} \leq C(\varepsilon)\left\|u_{t}(t, \cdot)\right\|_{m}^{m}+\varepsilon\|u(t, \cdot)\|_{p}^{p}, \\
\int \nabla u_{t}(t, x) \nabla u(t, x) d x \leq\|\nabla u(t, \cdot)\|_{2}\left\|\nabla u_{t}(t, \cdot)\right\|_{2} \\
\leq C(\varepsilon)\left\|\nabla u_{t}(t, \cdot)\right\|_{2}^{2}+\varepsilon\|\nabla u(t, \cdot)\|_{2}^{2} .
\end{gathered}
$$


Since

$$
\begin{aligned}
-R(u(t)) \geq & -R(u(t))+\delta(E(t)-E(0)) \\
\geq & \left(1-\frac{\delta}{p}\right)\|u(t, \cdot)\|_{p}^{p}+\frac{\delta}{2}\left\|u_{t}(t, \cdot)\right\|_{2}^{2} \\
& +\left(\frac{\delta}{2}-1\right)\left(\|\nabla u(t, \cdot)\|_{2}^{2}+\int V(x)|u(t, \cdot)|^{2} d x\right)-\delta E(0),
\end{aligned}
$$

we have

$$
\begin{aligned}
\frac{1}{2} J^{\prime \prime}(t) & +C(\varepsilon)\left\|u_{t}(t, \cdot)\right\|_{m}^{m}+C(\varepsilon)\left\|\nabla u_{t}(t, \cdot)\right\|_{2}^{2}+\varepsilon\|\nabla u(t, \cdot)\|_{2}^{2} \\
\geq & \left(1+\frac{\delta}{2}\right)\left\|u_{t}(t, \cdot)\right\|_{2}^{2}+\left(1-\frac{\delta}{p}-\varepsilon\right)\|u(t, \cdot)\|_{p}^{p} \\
& +\left(\frac{\delta}{2}-1-\varepsilon\right)\left(\|\nabla u(t, \cdot)\|_{2}^{2}+\int V(x)|u(t, \cdot)|^{2} d x\right)-\delta E(0),
\end{aligned}
$$

where the constant $\delta>2$ is chosen as follows.

Since $E(0)<d_{M}$, we choose the constant $\delta$ so that

$$
\frac{2 p d_{M}}{p d_{M}-(p-2) E(0)}<\delta<p .
$$

This guarantees that $\delta>2$. Then, using this choice and Lemma 4.2, we get

$$
\begin{aligned}
& \left(\frac{\delta}{2}-1\right)\left(\|\nabla u(t, \cdot)\|_{2}^{2}+\int V(x)|u(t, \cdot)|^{2} d x\right)-\delta E(0) \\
& \quad \geq\left(\frac{\delta}{2}-1\right) \frac{2 p}{p-2} d_{M}-\delta E(0) \geq 0
\end{aligned}
$$

If the constant $\delta$ is fixed, we choose the constant $\varepsilon$ such that

$$
C_{1}=1-\frac{\delta}{p}-\varepsilon>0 .
$$

Finally, using the inequality (4.6) and Lemmas 4.1 and 4.2 we have

$$
\begin{aligned}
J^{\prime \prime}(t) & +C(\varepsilon)\left\|u_{t}(t, \cdot)\right\|_{m}^{m}+C(\varepsilon)\left\|\nabla u_{t}(t, \cdot)\right\|_{2}^{2}+\varepsilon\|\nabla u(t, \cdot)\|_{2}^{2} \\
\geq & C_{1}\|u(t, \cdot)\|_{p}^{p} \\
\geq & C_{1}\left(\|\nabla u(t, \cdot)\|_{2}^{2}+\int V(x)|u(t, \cdot)|^{2} d x\right) \\
\geq & C_{1} \frac{2 p}{p-2} d_{M}
\end{aligned}
$$

where $C_{1}>0$. Since (4.3), integrating $(4.8)$ over $[0, t]$ we have

$$
J^{\prime}(t) \geq C_{1} \frac{p d_{M}}{p-2} t-C(\varepsilon) d+J^{\prime}(0)
$$


from which one concludes that there exists a $t_{1}$ such that $\left.J^{\prime}(t)\right|_{t=t_{1}}>0$. Hence, $J(t)$ is increasing for $t>t_{1}$ (which is the interval of existence). Since $R(u)<0$, there exists a $t_{2}$ such that $\|u(t, x)\|_{p}^{p}$ is increasing for $t>t_{2}$. When $t$ is large enough, the quantities $\left\|u_{t}(t, x)\right\|_{m}^{m}$ and $\|\nabla u(t, x)\|_{2}^{2}$ are small enough. Otherwise, assume that there is $t^{*}$ such that $\left\|u_{t}(t, x)\right\|_{m}^{m}>\left\|u_{t}\left(t^{*}, x\right)\right\|_{m}^{m}$ for all $t>t^{*}$. By integrating the inequality, we obtain a contradiction with (4.3) and $E(t) \geq 0$.

Thus in these cases, the quantity

$$
\begin{aligned}
& \left(1-\frac{\delta}{p}-\varepsilon\right)\|u(t, \cdot)\|_{p}^{p}+\left(\frac{\delta}{2}-1\right)\left(\|\nabla u(t, \cdot)\|_{2}^{2}+\int V(x)|u(t, \cdot)|^{2} d x\right)-\delta E(0) \\
& \quad-C(\varepsilon)\left\|u_{t}(t, \cdot)\right\|_{m}^{m}-C(\varepsilon)\left\|\nabla u_{t}(t, \cdot)\right\|_{2}^{2}-\varepsilon\|\nabla u(t, \cdot)\|_{2}^{2}
\end{aligned}
$$

will eventually become positive. Therefore for $t$ large enough, from (4.6) and (4.7) we have

$$
J^{\prime \prime}(t) \geq\left(1+\frac{\delta}{2}\right)\left\|u_{t}(t, \cdot)\right\|_{2}^{2}
$$

Using the Hölder inequality, we get

$$
J(t) J^{\prime \prime}(t) \geq \frac{2+\delta}{8}\left[J^{\prime}(t)\right]^{2}
$$

Since

$$
\left[J^{-\frac{\delta-6}{8}}(t)\right]^{\prime \prime}=-\frac{\delta-6}{8} J^{-\frac{\delta+10}{8}}(t)\left[J(t) J^{\prime \prime}(t)-\frac{2+\delta}{8}\left[J^{\prime}(t)\right]^{2}\right]
$$

from (4.10) we have $\left.J^{-\frac{\delta-6}{8}}(t)\right]^{\prime \prime} \leq 0$. Therefore $J^{-\frac{\delta-6}{8}}(t)$ is concave for sufficiently large $t$, and there exists a finite time $T^{*}$ such that

$$
\lim _{t \rightarrow T^{*}} J^{-\frac{\delta-6}{8}}(t)=0 .
$$

From assumption on $V(x)$, we obtain

$$
\int V(x)|u|^{2} d x \geq \bar{V} \int|u|^{2} d x
$$

Thus one get $T<\infty$ and

$$
\lim _{t \rightarrow T^{-}}\|u\|_{H}=\infty
$$

Now we complete the proof of (b1) of Theorem 2.5.

Next, we prove (b2) of Theorem 2.5.

From (2.7) and Lemma 4.1, we obtain $E(0)<S(Q)$. It follows that $u_{0}(x, t)$ satisfies

$$
R\left(u_{0}\right)=\int\left|\nabla u_{0}\right|^{2} d x+\int V(x)\left|u_{0}\right|^{2} d x-\int\left|u_{0}\right|^{p} d x>0,
$$


which will be proved by contradiction. If (4.11) is not true, then we have $R\left(u_{0}\right) \leq 0$. Thus there exists $0<\mu \leq 1$ such that $u_{0} \neq 0$ and

$$
R\left(\mu u_{0}\right)=\mu^{2}\left(\int\left|\nabla u_{0}\right|^{2} d x+\int V(x)\left|u_{0}\right|^{2} d x\right)-\mu^{p} \int\left|u_{0}\right|^{p} d x=0,
$$

which implies $\mu u_{0} \in M$.

On the other hand, for $0<\mu \leq 1, u_{0} \in K_{2}$ and (2.3) yield

$$
S\left(\mu u_{0}\right)<\mu^{2}\left(\int\left|\nabla u_{0}\right|^{2} d x+\int V(x)\left|u_{0}\right|^{2} d x\right) \leq \frac{p-2}{2 p} \int|Q|^{p} d x=S(Q),
$$

which is contradictory to Lemma 3.1.

Therefore, by $R\left(u_{0}\right)>0$ and Lemma 4.1, we have $R(u)>0$ and $E(t) \leq E(0)<S(Q)$. Thus

$$
E(t)-\frac{1}{p} S(u) \leq E(0)
$$

namely

$$
\frac{1}{2} \int\left|u_{t}\right|^{2} d x+\frac{p-2}{2 p}\left(\int|\nabla u|^{2} d x+\int V(x)|u|^{2} d x\right) \leq E(0) .
$$

Therefore we have established the bound of $u(x, t)$ in $H^{1}$ for $t \in[0, T)$ and thus the solution $u(x, t)$ of $(1.1)$ exists globally on $t \in[0, \infty)$.

From (4.11), $E(0)<S(Q)$ and Lemma 3.1, we have the estimate (2.8).

Thus, we complete the proof of Theorem 2.5.

\section{Competing interests}

The author declares that they have no competing interests.

Received: 27 October 2013 Accepted: 27 May 2014 Published online: 10 September 2014

\section{References}

1. Gazzola, F, Squassina, M: Global solutions and finite time blow up for damped semilinear wave equations. Ann. Inst. Henri Poincaré, Anal. Non Linéaire 23, 185-207 (2006)

2. Soffer, A, Weinstein, Ml: Resonance radiation damping and instability in Hamiltonian nonlinear wave equations. Invent. Math. 136, 9-74 (1999)

3. Levine, HA: Instability and nonexistence of global solutions to nonlinear wave equations of the form $P u_{t t}=-A u+F(u)$. Trans. Am. Math. Soc. 192, 1-21 (1974)

4. Georgiev, V, Todorova, G: Existence of a solution of the wave equation with nonlinear damping and source terms J. Differ. Equ. 109, 295-308 (1994)

5. Todorova, G: Cauchy problems for a nonlinear wave equations with nonlinear damping term. Nonlinear Anal. 41, 891-905 (2000)

6. Ikehata, R: Some remarks on the wave equations with nonlinear damping and source terms. Nonlinear Anal. 27, 1165-1175 (1996)

7. Todorova, G: Cauchy problem for a nonlinear wave equation with nonlinear damping and source terms. C. R. Acad. Sci., Sér. 1 Math. 326, 191-196 (1998)

8. Zhang, J: Stability of standing waves for nonlinear Schrödinger equations with unbounded potentials. Z. Angew. Math. Phys. 51, 498-503 (2000)

9. Huang, WY, Lai, SY, Zhang, J: Sharp conditions of global existence for the nonlinear Klein-Gordon equation. Acta Math. Sin. 54, 435-442 (2011)

10. Pucci, P, Serrin, J: Global nonexistence for abstract evolution equations with positive initial energy. J. Differ. Equ. 150 203-214 (1998)

11. Liu, WJ: Global existence, asymptotic behavior and blow-up of solutions for coupled Klein-Gordon equations with damping terms. Nonlinear Anal. 73, 244-255 (2010)

12. Zhang, ZY, Miao, XJ: Global existence and uniform decay for wave equation with dissipative term and boundary damping. Comput. Math. Appl. 59, 1003-1018 (2010) 
doi:10.1186/s13661-014-0144-0

Cite this article as: Huang: Standing waves and global existence for nonlinear wave equations with potential, strong, and nonlinear damping terms. Boundary Value Problems 2014 2014:144.

Submit your manuscript to a SpringerOpen ${ }^{\circ}$ journal and benefit from:

- Convenient online submission

- Rigorous peer review

- Immediate publication on acceptance

- Open access: articles freely available online

- High visibility within the field

- Retaining the copyright to your article

Submit your next manuscript at $\gg$ springeropen.com 